\title{
Chandra Observations of Old Novae
}

\author{
Koji Mukai $^{*}$, Marina Orio ${ }^{\dagger}$, Fred Ringwald ${ }^{* *}$ and Martin Still* \\ ${ }^{*}$ Code 662, NASA Goddard Space Flight Center, Greenbelt, MD 20771, USA (also Universities \\ Space Research Association) \\ $\dagger^{\dagger}$ Astronomy Dept., Univ. Wisconsin, 475 N. Charter Str, Madison WI 53706, USA (also \\ Osservatorio Astronomico di Torino) \\ ${ }^{* *}$ Department of Physics, California State University, Fresno, 2345 E. San Ramon Ave., M/S \\ MH37, Fresno, CA 93740-8031, USA
}

\begin{abstract}
We present highlights of Chandra observations of two old novae, DQ Her and V603 Aql, with the main aim of improving our understanding of the underlying binaries decades after their respective nova eruptions. In DQ Her, we find a partial X-ray eclipse; it is likely that we observe photons scattered in an accretion disk wind. The X-ray spectrum of V603 Aq1 suggests an origin in multi-temperature plasma; while the low energy lines suggest modest density, the $6.4 \mathrm{keV} \mathrm{Fe} \mathrm{K \alpha}$ line suggests that the hard continuum arises in a compact emission region. We also report on our searches for nebular X-ray emission around these old novae.
\end{abstract}

\section{INTRODUCTION}

X-ray observations of old novae are useful in selecting magnetic systems, in constraining the total accretion rate, and potentially in inferring the abundances of the accreting and/or ejected materials. With the increasing capability of X-ray satellites, we can now observe fainter systems or obtain detailed light curves and spectra of brighter systems. Here we present our Chandra observations of DQ Her and V603 Aq1. The former is Xray faint, while the latter is among the X-ray brightest old novae known, although both were among the brightest novae of the 20th century.

\section{DQ HER}

DQ Her (Nova Herculis 1934, at an estimated distance of $\sim 560 \mathrm{pc}$; [1]) is the prototype of a subclass of magnetic CVs, "Intermediate Polars" (IPs) or "DQ Her type systems" [2]. Unlike most members, however, DQ Her is not a strong X-ray source as seen from Earth. It was undetected with Einstein [3], with an upper limit of $3 \times 10^{30} \mathrm{ergs} \mathrm{s}^{-1}$. Since DQ Her is a deeply eclipsing system in the optical, its white dwarf (the presumed primary X-ray emission site) is likely hidden from our view at all times. DQ Her, however, was detected with ROSAT at $\sim 4.0 \times 10^{30} \mathrm{ergs} \mathrm{s}^{-1}$ [4]. A deep eclipse was not observed, hence these detected X-rays are not from the immediate vicinity of the white dwarf. So what is the origin of the observed X-rays?

We observed DQ Her with Chandra ACIS-S in imaging mode (i.e., without a grating) from 2001 July 26 13:00 UT - July 27 02:31 UT and again from 2001 July 29 17:09 UT

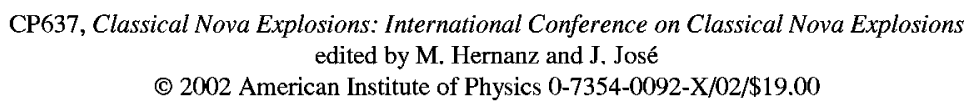



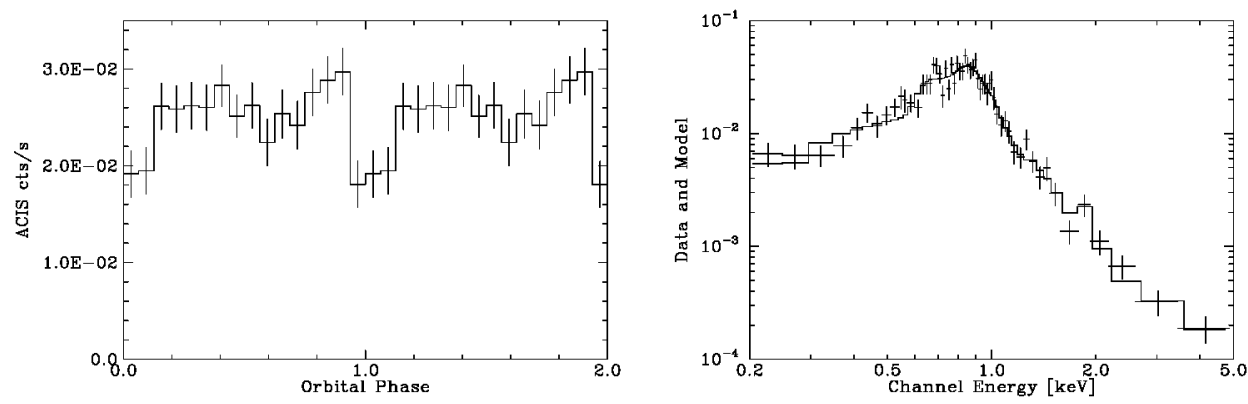

FIGURE 1. (Left) The X-ray light curve of DQ Her, folded on the orbital period (two cycles are shown for clarity). (Right) The X-ray spectrum of DQ Her, as observed with Chandra ACIS-S, plotted with a 2-component model (power law plus a $\mathrm{kT} \sim 0.6 \mathrm{keV}$ thermal plasma model) folded with the ACIS-S response.

- July 30 02:27 UT, for a total integration time of $69 \mathrm{ksec}$. We detect a moderately strong $\left(0.025 \mathrm{cts} \mathrm{s}^{-1}\right.$, or $\left.\sim 3 \times 10^{30} \mathrm{ergs} \mathrm{s}^{-1}\right)$ point source at the optical position of DQ Her in these observations. The light curve, folded on the orbital ephemeris of [5], clearly shows a partial eclipse, lasting just over 0.1 in orbital phase and about $30 \%$ deep. The spectrum shows a bump around $0.8-1.0 \mathrm{keV}$, characteristic of plasma emission with lines of $\mathrm{O}$, $\mathrm{Ne}$, and $\mathrm{Fe}$ at these energies (Figure 1). The partial eclipse is a signature of an extended emission region. Material that is $\sim 11$ white dwarf radii above the orbital plane should remain uneclipsed, given the known system geometry, suggesting a vertical extent at least this much. These observations are consistent with the idea that the observed Xrays have been scattered in an accretion disk wind, similarly to OY Car in superoutburst [6]. The presence of an accretion disk wind in DQ Her has been inferred from UV observations [7], even though DQ Her lacks a boundary layer. The fact that there is a significant partial X-ray eclipse in DQ Her makes it different from other wind-scattered X-ray sources such as OY Car and UX UMa [8], and may be an indication of differences in their wind structures.

\section{V603 AQL}

V603 Aql (Nova Aquilae 1918) is among the X-ray brightest old novae both in intrinsic luminosity (in excess of $10^{32} \mathrm{ergs} \mathrm{s}^{-1}$ ) and in terms of flux at Earth [9]. We therefore observed V603 Aq1 with Chandra HETG with ACIS-S from 2001 April 19 17:36 UT April $2011: 53$ UT for a total of $64 \mathrm{ksec}$, to obtain the highest quality X-ray spectrum of an old nova to date.

There have been claims that this is a magnetic system of the IP type, based on polarimetry [10] and on X-ray photometry [11]. However, the former was based on a method which is, for variable sources, susceptible to false detections; on the other hand, [12] established a strict upper limit using a variable-star-safe instrument. Here we examine the claim of X-ray periodicity (Figure 2), using 1 st order (dispersed) photons in the Chandra HETG data which were taken without interruption (0th order, undispersed, 


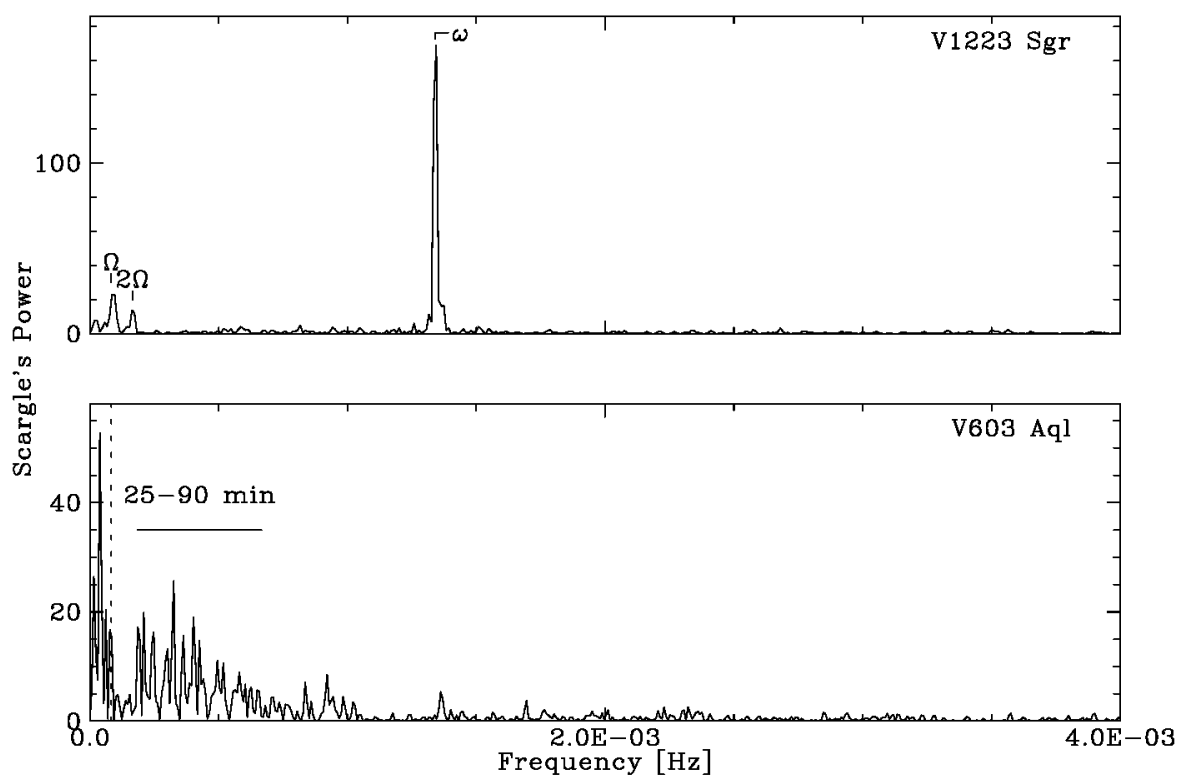

FIGURE 2. Power spectra of the IP, V1223 Sgr (top) and the old nova, V603 Aql (bottom), both from continuous Chandra grating observations. The former shows strong peaks at the orbital and spin periods, whereas the latter shows a broad envelope of excess power: the observed X-ray variability is not periodic.

image suffers from a significant pile-up which makes it far less useful for timing analysis). As a comparison, the Chandra HETG power spectrum of V1223 Sgr [13], a typical IP, shows prominent peaks at the orbital and spin periods, and in some harmonics, with little power at other frequencies. In contrast, the power spectrum of V603 Aq1 contains a number of peaks, roughly in the $20 \mathrm{~min}-1 \mathrm{hr}$ range. It is highly unlikely that any combinations of harmonics and sidebands can explain all the peaks, given a single underlying clock. We therefore conclude that V603 Aql shows a strong variability, but not a periodicity, and that there is no evidence to date that it is an IP.

The average spectrum of V603 Aql is rich in emission lines; the simultaneous presence of emission lines of $\mathrm{Fe}, \mathrm{Si}, \mathrm{Mg}, \mathrm{Ne}$, and $\mathrm{O}$ is a direct evidence for the multitemperature nature of the X-ray emitting plasma. In Figure 3, we show details of selected regions of the spectrum. With the superior spectral resolution of Chandra HETG, we have been able to resolve the He-like triplets of $\mathrm{Ne}$ IX and $\mathrm{Mg}$ XI. The presence of the forbidden ( $\mathrm{f}$ ) component in the latter (and perhaps also the former) sets a limit of the density of the line emitting region at $n_{e} \leq 10^{13} \mathrm{~cm}^{-3}$. At the $\mathrm{Fe} \mathrm{K}$ region of the spectrum, the resolution of HETG is insufficient to resolve the He-like triplets; what we see are the $6.4 \mathrm{keV}$ fluorescent, $6.7 \mathrm{keV} \mathrm{He}$-like, and $6.97 \mathrm{keV} \mathrm{H}$-like components. The $\mathrm{H}-$ like component, from $\mathrm{kT} \sim 10 \mathrm{keV}$ plasma, is present but significantly weaker than the He-like component $(\mathrm{kT} \sim 5 \mathrm{keV})$. The fluorescent component has an equivalent width of $\sim 150 \mathrm{eV}$, consistent with reflection from the white dwarf surface that subtends $2 \pi$ steradians. This implies that the hard X-ray continuum originates in a compact emission 

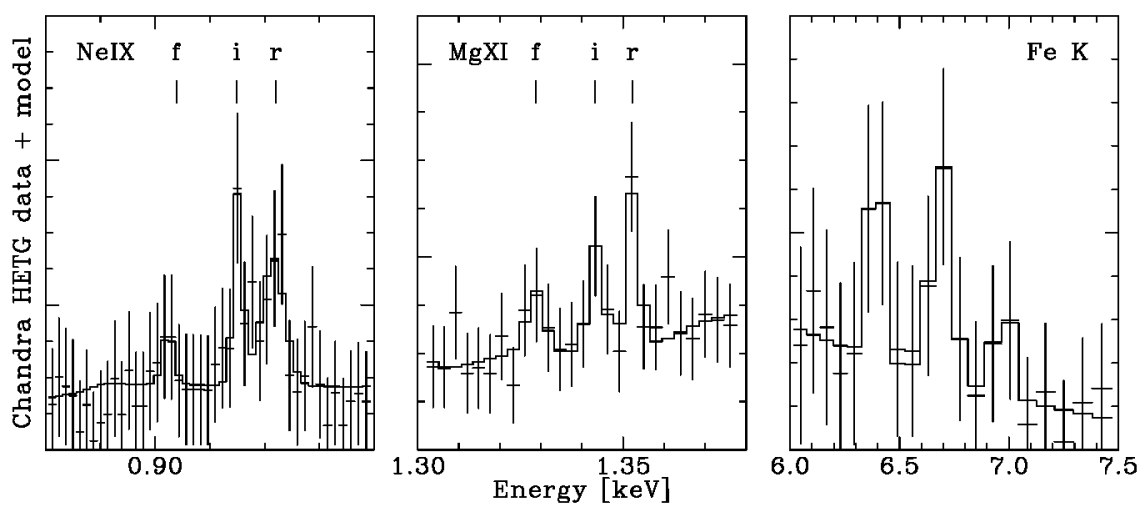

FIGURE 3. Selected X-ray emission lines of V603 Aql. The left two panels show He-like triplets of $\mathrm{Ne}$ IX and Mg XI while the right panel shows the Fe K line ( $\sim$ neutral, He-like and H-like).

region, whereas the lower energy lines may originate in a more extended structure.

\section{A SEARCH FOR NEBULAR X-RAY EMISSIONS}

Finally, the superior imaging resolution of Chandra allows a sensitive search for extended emission, such as that seen around GK Per [14]. In Figure 4, we plot the observed radial profile of the Chandra image of DQ Her, and the best-fit model based on the known point-spread function (the fit is strongly constrained by the first few points, which are not shown). We detect an excess of counts 5-10 arcsec away, most prominently at the lowest energies. This excess is most prominent NE of DQ Her itself, and is not consistent with a point source. A comparison with ground-based images of the nova shell [15] suggests a coincidence with an [NII] knot.

Our search for a similar feature around V603 Aql has been inconclusive. This is because we used a grating, which complicates the background and the point-spreadfunction calibration; and because the central source is much brighter than in DQ Her.

\section{CONCLUSIONS}

The majority of X-rays from DQ Her appear to be scattered in the accretion disk wind. Despite its well-credentialed magnetic nature, DQ Her resembles high accretion rate, non-magnetic CVs seen at high inclination. X-ray data may be useful more as a probe of the wind than of the accretion flow immediately around the white dwarf in this system. A small number of X-ray photons $\left(\sim 30\right.$, a few times $\left.10^{28} \mathrm{ergs} \mathrm{s}^{-1}\right)$ appear to be from the shell around DQ Her.

The X-ray spectrum of V603 Aq1 is rich in lines and may contain enough clues to advance our understanding of accretion onto the white dwarf. However, we have not yet 


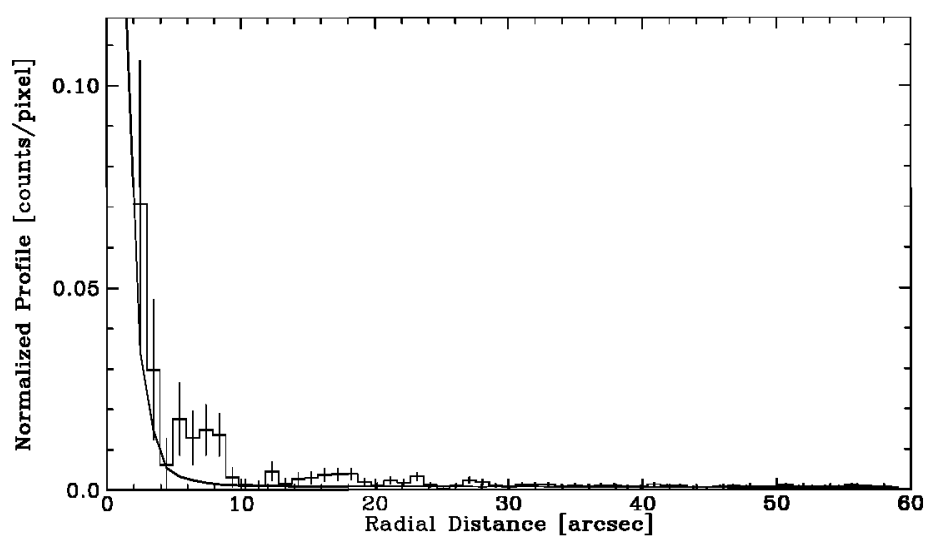

FIGURE 4. The radial profile of the Chandra ACIS-S X-ray image of DQ Her in the $0.2-0.5 \mathrm{keV}$ band. The data are plotted as a histogram with errors, while the best-fit model (for $0.3 \mathrm{keV}$ monochromatic $\mathrm{X}$-rays observed on-axis) are plotted as a solid line.

learned how to interpret these clues. It may serve as a template for the studies of nonmagnetic CVs in general (previous claims that V603 Aq1 is magnetic were premature, and quite possibly erroneous).

We have presented a flavor of what X-ray observations of quiescent old novae can do. Observations of additional novae with Chandra and XMM-Newton should be encouraged.

\section{REFERENCES}

1. Herbig, G.H., and Smak, J.I, Acta Astron. 42, 17-28 (1992).

2. Patterson, J., PASP 106, $209-238$ (1994).

3. Córdova, F.A., Mason, K.O., and Nelson, J.E., Ap.J. 245, 609-617 (1981).

4. Silber, A.D., Anderson, S.F., Margon, B. \& Downes, R.A., Ap.J. 462, 428-438 (1996).

5. Zhang, E., Robinson, E.L., Stiening, R.F., and Horne, K., Ap.J. 454, 447-462 (1995).

6. Mauche, C.W., and Raymond, J.C., Ap.J. 541, 924-936 (2001).

7. Córdova, F.A., and Mason, K.O, Ap.J. 290, 671-682 (1985).

8. Wood, J.H., Naylor, T., and Marsh, T.R., Mon. Not. R. astr. Soc. 274, 31-36 (1995).

9. Becker, R.H., and Marshall, F.E., Ap.J.Lett. 244, L93-L95 (1981).

10. Haefner, R., and Metz, K., Astron. Ap. 145, 311-320 (1985).

11. Udalski, A., and Schwarzenberg-Czerny, A., Acta Astron. 39, 125-138 (1989).

12. Naylor, T., Koch-Miramond, L., Ringwald, F.A., and Evans, A., Mon. Not. R. astr. Soc. 282, 873-876 (1996).

13. Mukai, K., Kallman, T., Schlegel, E., Bruch, A., Handler, G., and Kemp, J., "Chandra HETG Observation of the Magnetic Cataclysmic Variable V1223 Sagittarii," in New Century of X-ray Astronomy, edited by H. Inoue and H. Kunieda, ASP Conf. Ser. 251, San Francisco, 2001, pp. 90-93.

14. Balman, $\mathrm{S}$., this volume.

15. Slavin, A.J., O’Brien, T.J., and Dunlop, J.S., Mon. Not. R. astr. Soc. 276, 353-371 (1995). 
Copyright $@ 2002$ EBSCO Publishing 
Copyright of AIP Conference Proceedings is the property of American Institute of Physics and its content may not be copied or emailed to multiple sites or posted to a listserv without the copyright holder's express written permission. However, users may print, download, or email articles for individual use. 